\title{
A Variational Approach for Multi-valued Velocity Field Estimation in Transparent Sequences
}

\author{
Alonso Ramírez-Manzanares ${ }^{1}$, Mariano Rivera ${ }^{1}$, Pierre Kornprobst ${ }^{2}$, \\ and François Lauze ${ }^{3}$ \\ ${ }^{1}$ Centro de Investigacion en Matematicas A.C., AP 402, Guanajuato, Gto., 36000, \\ Mexico \\ \{alram,mrivera\}@cimat.mx \\ 2 INRIA, Odyssée Lab., 2004 route des Lucioles BP 93, 06902 Sophia Antipolis, \\ France \\ Pierre.Kornprobst@sophia.inria.fr \\ ${ }^{3}$ Nordic Bioscience Imaging A/S, DK-2370 Herlev, Denmark \\ francois@nordicbioscience.com
}

\begin{abstract}
We propose a variational approach for multi-valued velocity field estimation in transparent sequences. Starting from existing local motion estimators, we show a variational model for integrating in space and time these local estimations to obtain a robust estimation of the multi-valued velocity field. With this approach, we can indeed estimate some multi-valued velocity fields which are not necessarily piecewise constant on a layer: Each layer can evolve according to non-parametric optical flow. We show how our approach outperforms some existing approaches, and we illustrate its capabilities on several challenging synthetic/real sequences.
\end{abstract}

Keywords: Variational approaches, transparent motion, multi-valued velocity fields, model competition.

\section{Introduction}

There exists a very wide literature on apparent motion estimation, also called optical flow (OF), due to the number of applications that require motion estimation, and the complexity of the task. Motion estimation methods often rely on an intensity conservation principle and on spatial or spatiotemporal regularity constraints. The simplest conservation principle states that the intensity of a point remains constant along its trajectory. Although widely used, this principle is not satisfied in several real situations which include changing illuminance conditions, specularities, and multiple motions, as it is for instance the case in transparency.

Transparency can be modeled as a linear superposition of moving layers, meaning addition of layer intensities, or a generalized one 11. A simple superposition model has been introduced by Burt et al in 2, from which they derive 
an iterative three frame algorithm for estimating two motions. A more thorough study and extension of this idea is proposed in $[3$, providing a frequency domain interpretation and explaining a mechanism of "dominant velocity extraction". It is used by Irani and Peleg in 4 (see also [5]). Shizawa and Mase 6 617/8 explore a frequency domain, total least squares formulation of the multiple motion problems, providing a 2-fold OF constraint equation, a closed-form formula is proved for two motions, but the problem becomes rapidly more complicated for higher orders. Liu et al. use it in [9] with Hermite polynomial based differentiation filters, and the authors check for the presence of single or multiple motions. Darell and Simoncelli [10 "dualize" this constraint in order to construct some Fourier "donuts" that respond to one or more velocities. Mota et al. have extended these ideas in [1] and Mühlich and Aach have proposed an algebraic framework based on homogeneous components of symmetric algebras in [12. The nonlinear form of the 2-fold OF constraint provides what one may call the 2-fold displaced frame difference equation, it can be extended to more than two motions to derive a block-matching approach to the multiple motion problem [13. In summary, this class of approach is based on a single higher order constraint designed to "react" to multiple motions.

A spatial regularization in the framework of Markov Random Fields of the block matching solution is proposed in 14, in order to promote smooth solutions. However finding the global solution of the energy minimization method results in a computationally expensive minimization, because of the use of a field of binary indicator variables. Similarly, in [15] a parametric variant of the above block matching was applied in a layered approach for transparent X-Ray sequences (where the integration of material density produces transparent sequences [16]). The method segments and estimates the OF by alternately apply IRLS and ICM methods in order to compute both layer indicator variables and velocities.

Another important class of approaches is based on multiple low order motion constraints, designed to detect single motions. In the robust statistics approach of Black and Anandan [17, the image plane is assumed to be partitioned into regions, each one corresponding to a parametric motion model. The motion parameters are then assumed to represent the motion of two layers that cover the entire image plane. The layers are recovered by an iterative parameter/region estimation and by a nulling process. Mixture models for multiple motion computation have been introduced by Jepson and Black [18. One assumes that the motion in layers can be explained by up to $N$ parametric motion fields by computing the best mixture and motion parameters, usually done by using EM-like algorithms. Ju et al 19] propose a model, in which, multilayered affine models are defined on small rectangular image patches (bones), and an inter-patch term (skin) introduces a regularization effect in the model parameters estimation. Then layer ownerships and affine model parameters are computed within a robust estimation framework by using an EM-algorithm. Black et al 20 compute a set of membership weights in order to link layers with regions. Although the method captures the changes in illumination, it does not allow the computation of the OF of moving transparencies. Weiss and Adelson [21] and Rivera et al 22] 
proposed EM-based approaches for computing different layered motion models. They use as prior knowledge the smooth feature of the velocities. The solution is given by a field of layer probabilities. Both methods produce pixel-wise unimodal solutions (single motions) since they use a distance measure for single motions as well as entropy controls. For more details about the different types of constraints and proposed approaches, we refer the reader to 23 .

In this work we propose an approach based on local detectors sensitive to one or more velocities. We observed that the responses of these detectors usually provide a very local, noisy and somewhat too complex description of the velocities (more velocities than actually present may be detected at a given location). So there is a need for integration and regularization of this local information, which can be naturally performed with the framework of variational approaches.

The paper is organized as follows. Section 2 describes the proposed framework based on a finite sampling of the space of velocities and states a discrete variational model to handle multiple motions. Our approach encodes prior knowledge about the OF smoothness and the expected, relatively small, number of motions per pixel (one or two). The method performance is illustrated in Section 3 , on synthetic, synthesized realistic and real sequences. We conclude and present future work in Section 4 .

\section{From Local to Global by a Variational Model}

Let us introduce the main notations. The function $f:(\mathbf{x}, t) \in \Omega \times\{0, \ldots, T\} \rightarrow R$ denotes the input sequence, defined as a volume over space and time. Let us now define a finite sampling of the velocity space: We consider $N$ vectors

$$
\left\{u_{1}, \ldots, u_{N}\right\} \text {, with } u_{i}=\left(u_{i 1}, u_{i 2}\right)^{T},
$$

describing the set of possible velocities. Our goal will be to determine what is the likelihood of having velocity $u_{i}$ at a given position. To do so, one need an initial local estimate of this likelihood. Let us assume that we know a function $\left.d\left(u_{i}, r\right) \in \mathbb{R}^{+}\right|_{i=1 \ldots N}$ which describe at each position $r=(\mathbf{x}, t)$ whether the velocity $u_{i}$ can explain locally the apparent motion (characterized by $d\left(u_{i}, r\right) \approx$ 0 ) or not (characterized $\left.d\left(u_{i}, r\right) \gg 0\right)$. In general, $d$ can be implemented as a norm (or quasi-norm) over a similarity operator, and we refer to Section 2.1 for more details.

So, given a sequence $f$ and the local motions estimations $\left.d\left(u_{i}, r\right)\right|_{i=1 \ldots N}$, our goal is to propose an approach which integrates this local information in order to obtain a global and robust velocity estimation. This integration is necessary to deal with noisy sequences but also sequences with complex motions such as transparent motion.

\subsection{How to Estimate Local Velocity Information?}

Two well known similarity operators satisfy these requirements for the single motion case: The non-linear difference (Correlation-based)

$$
M_{\mathcal{C}}^{(1)}\left(u_{i}\right) f(\mathbf{x}, t) \stackrel{\text { def }}{=} f(\mathbf{x}, t)-f\left(\mathbf{x}-u_{i}, t-1\right),
$$


and its linearized version (Differential-based)

$$
M_{\mathcal{D}}^{(1)}\left(u_{i}\right) f\left(x_{1}, x_{2}, t\right) \stackrel{\text { def }}{=}\left(u_{i 1} \frac{\partial}{\partial x_{1}}+u_{i 2} \frac{\partial}{\partial x_{2}}+\frac{\partial}{\partial t}\right) f\left(x_{1}, x_{2}, t\right),
$$

where the superscript indicates the number of displacements that these operators (and the following ones) take into account. Following Shizawa and Mase [8], one can define an operator for two velocities as the composition

$$
M_{\mathcal{D}}^{(2)}\left(u_{i}, u_{j}\right) f(\mathbf{x}, t) \stackrel{\text { def }}{=} M_{\mathcal{D}}^{(1)}\left(u_{i}\right) M_{\mathcal{D}}^{(1)}\left(u_{j}\right) f(\mathbf{x}, t)
$$

where products $\frac{\partial}{\partial r} \frac{\partial}{\partial s}$ are naturally expanded as $\frac{\partial^{2}}{\partial r \partial s}$. Composing instead the nonlinear correlation operators $M_{\mathcal{C}}^{(1)}$ provides the nonlinear operator for two velocities

$M_{\mathcal{C}}^{(2)}\left(u_{i}, u_{j}\right) f(\mathbf{x}, t) \stackrel{\text { def }}{=} f(\mathbf{x}, t)-f\left(\mathbf{x}-u_{i}, t-1\right)-f\left(\mathbf{x}-u_{j}, t-1\right)+f\left(\mathbf{x}-u_{i}-u_{j}, t-2\right)$,

that corresponds to distance reported in [13].

We introduce here the general mechanism we have used in order to select the local velocity descriptors $d\left(u_{i}, r\right)$ from multiple motion operators. Given an integer $k \in[1, N]$, let us assume that we have a family of " $k$ velocities probe" operators

$$
\mathbf{M}=\left\{M^{(k)}\left(u_{i_{1}}, \ldots, u_{i_{k}}\right), \quad 1 \leq i_{1}<\cdots<i_{k} \leq N\right\}
$$

where $M^{(k)}\left(u_{i_{1}}, \ldots, u_{i_{k}}\right) f(r) \approx 0$ if the velocity vectors $u_{i_{1}}, \ldots, u_{i_{k}}$ explain the motion of the image sequence $f$ at the position $r$. We build them by either cascading $k$ correlation based filters, such a probe will be denoted in general $M_{\mathcal{C}}$, or $k$ differential-based ones, these ones will be denoted $M_{\mathcal{D}}$. Then for each vector $u_{i}$, let us consider the subset $\mathbf{M}_{u_{i}}$ of all the operators involving $u_{i}$ and define

$$
d_{\mathcal{C}}\left(u_{i}, r\right)=\min _{M_{\mathcal{C}} \in \mathbf{M}_{u_{i}}} \frac{1}{k} \sum_{s \in W_{r}}\left(M_{\mathcal{C}} f(s)\right)^{2}
$$

and

$$
d_{\mathcal{D}}\left(u_{i}, r\right)=\min _{M_{\mathcal{D}} \in \mathbf{M}_{u_{i}}} \frac{1}{k} \sum_{s \in W_{r}}\left(M_{\mathcal{D}} f(s)\right)^{2},
$$

where $W_{r}$ is a $3 \times 3$ spatial window center at $r$. In the present work, we used as input distances (11) and (2) for different experiments, showing the general framework feature of our proposal as is explained in the following. Note that because of the series Taylor's approximation, distance (1) is more suitable than (21) for long displacements.

\subsection{Global Motion Integration Via a Variational Approach}

Let us define the function $r=(\mathbf{x}, t) \mapsto \alpha_{i}(r) \in[0,1]$, which will correspond to the probability that velocity $u_{i}$ explains the apparent motion at the spatiotemporal position $r$. We define the unknowns of the problem as the vector valued field $\alpha$ :

$$
\alpha(r)=\left(\alpha_{1}(r), \ldots, \alpha_{N}(r)\right), \quad \alpha_{i}(r) \in[0,1] \quad \forall r \in \Omega \times\{0, \ldots T\} .
$$


Note that, although each component of $\alpha(r)$ can be interpreted as a probability, $\alpha(r)$ is not a probability measure (as in [21|22]) in the sense that the sum of its components is not constrained to be one. If two motions $u_{i}$ and $u_{j}$ are present at a particular pixel position, then we expect that $\alpha_{i}(r) \approx \alpha_{j}(r) \approx 1$. Inversely, the velocity field at a position $r$ can be extracted from $\alpha(r)$ by keeping the velocity $u_{i}$ with high value of $\alpha_{i}(r)$.

In order to estimate the global multi-valued velocity field from the local data $d\left(u_{i}, r\right)$, we propose to minimizes the cost function $\mathcal{E}(\alpha)$ defined by

$$
\begin{aligned}
\mathcal{E}(\alpha)=\sum_{r}\left\{\sum_{i} d\left(u_{i}, r\right) \alpha_{i}^{2}(r)\right. & \\
& +\frac{\lambda_{s}}{2} \sum_{s: s \in \mathcal{N}_{r}} \sum_{i} w_{i}(r, s)\left[\alpha_{i}(r)-\alpha_{i}(s)\right]^{2} \\
& \left.+\lambda_{c}\left[c \bar{\alpha}^{2}(r)-\sum_{i} \alpha_{i}^{2}(r)\right]\right\},
\end{aligned}
$$

subject to the constraints $\alpha_{i}(r) \in[0,1]$ for all $i$, with $\bar{\alpha}(r) \stackrel{\text { def }}{=} \frac{1}{N} \sum_{i} \alpha_{i}(r)$, $w_{i}(r, s)$ are some diffusion weight defined in the sequel, $\mathcal{N}_{r} \stackrel{\text { def }}{=}\{s: r, s \in \Omega \times$ $[0, T],\|r-s\|<2\}$ is the spatiotemporal neighborhood of the $r$ position, and $c, \lambda_{s}, \lambda_{c}$ are some positive constants. Let us now comment the different terms of this energy.

Term (3): Attach Term. This term links the input (the functions $d$ 's) to the unknown $\alpha$. For computing the presence of the $i$-th model, we use an approach related with the outlier rejection method 24 and with the EM formulation 201212219]. Minimizing term (3) w.r.t. $\alpha_{i}(r)$ produces $\alpha_{i}(r)$ close to 0 for high $d\left(u_{i}, r\right)$ values, indicating in this way that such a motion model is not likely at position $r$. Otherwise, the $\alpha_{i}(r)$ is free and its value is established by the next terms and the bound constraint.

Term (4): Spatial Regularization. Local information is integrated through this regularization term. At a given location $r$, we minimize the difference between vector $\alpha(r)$ and all the vectors $\alpha(s)$ in the neighborhood $\mathcal{N}_{r}$. Because our indicator variables are real valued, we can use differentiable potentials with their well-known algorithmic advantages. We use the approach presented in [25]. The smoothing process is controlled by directional fixed weights, $w_{i}(r, s)=$ $\left((s-r)^{T} \overline{\mathbf{I}}_{i}(s-r)\right) /\|s-r\|^{4}$, generated from the $i$-th tensor associated to the $i$-th velocity model: $\overline{\mathbf{I}}_{i}=\gamma I_{d}+U_{i} U_{i}^{T}$, where $I_{d}$ is the identity matrix, $\gamma=0.1$ and $U_{i}=\left[u_{i 1}, u_{i 2}, 1\right]^{T} /\left\|\left[u_{i 1}, u_{i 2}, 1\right]\right\|$ is a homogeneous-coordinate unit vector. For small $\gamma$ values (as the one proposed here) these weights promote a strong smoothness along the $i$-th velocity direction. The arbitrarily fixed 4 -th power of the distance restricts the spatial influence of the smoothness term, see also [23. As a consequence piece-wise smooth OFs are recovered with well-defined boundaries along the velocity model (see Figure 3 ). 
Term (5): Intra-Model Competition. Our aim is to detect multiple simultaneous motions (transparent motions), thus we may have problems at sites where multiple spurious matches are locally detected, for example in homogeneous regions, where $d\left(u_{i}, r\right) \approx 0$ for many (maybe all) velocities. For this reason we need a mechanism to eliminate spurious models (i.e. to cancel some of the components of $\alpha(r))$ and to promote the valid ones: We want to recover almost binary solutions (similar to the entropy-control potentials, see for example the Shannon one [21] or the Gini one [22]). In our case, we use potential (5) because $\alpha(r)$ is not a probability measure and also because this potential is well adapted for recovering multimodal solutions. The parameter $c$ is a tuning parameter related to the number of switched-on models. To understand the potential's behavior, we note that the first term penalizes the number of switched-on models while the second term promotes the "switching on" of models and avoids the trivial null solution $\alpha(r)=0$. Hence for a fixed mean value (controlled by the first term) the second term prefers highly contrasted solutions. Note that (5) can be tuned such that for a given $c$ value, a multimodal solution has lower energy than an unimodal one or conversely. That makes an important distinction with respect to entropy based measures that always have lower energy for unimodal solutions 21/22. Additionally, our proposed quadratic potential is easily differentiable and therefore simple minimization algorithms can be used as explained below.

Algorithmic Details. The cost function $\mathcal{E}(\alpha)$ is quadratic and can be minimized by solving the linear system $\partial \mathcal{E}(\alpha) / \partial \alpha_{i}(r)=0, \quad \forall i, \forall r$, with the constraints $\alpha_{i}(r) \in[0,1]$. We use a Gauss-Seidel algorithm. Given an estimate $\alpha_{i}^{n}$, we iterate until convergence:

$$
\alpha_{i}^{n+1}(r)=\frac{\lambda_{s} \sum_{s \in \mathcal{N}_{r}} w_{i}(r, s) \alpha_{i}^{n}(s)-c \lambda_{c} \bar{\alpha}^{n}(r)}{d\left(u_{i}, r\right)+\lambda_{s} \sum_{s \in \mathcal{N}_{r}} w_{i}(r, s)-\lambda_{c}} .
$$

The bound constraints on $\alpha_{i}(r)$ are then enforced by projecting non-feasible values to bounds at each iteration. We note that for obtaining a smooth algorithm convergence, it was important to keep fixed the mean of the previous iteration, $\bar{\alpha}^{n}(r)$, for updating the current $\alpha(r)$ vector. This can be seen as an over-relaxation strategy. We initially set $\alpha_{i}^{0}(r)=0.5, \forall i, r$.

A Deterministic Annealing strategy in the $\lambda_{c}$ parameter introduces the intramodel competition only until an approximate solution with valid representative models have predominant $\alpha_{j}(r)$ values: For each iteration $k=1,2, \ldots, n$, we set $\lambda_{c}^{(k)}=\lambda_{c} a_{k}$, where $\lambda_{c}$ is the chosen contrast level and $a_{k}=0.1+0.9(1-$ $\left.0.95^{(100 k / n)}\right)$ is a factor that increases to 1 in approximately $80 \%$ of the total iterations. Results are sensitive to the annealing speed of $\lambda_{c}$ : Premature increment could lead to an incorrect solution. Nevertheless, we used the same annealing scheduling in all our experiments. The large value $\lambda_{s}$ eliminates noise but one too large blurred the motion boundaries. We used $\lambda_{s} \in[50,100]$ for an adequate noise reduction. Parameter $c=1$ performs well for most noise-free synthetic sequences. For noise-contaminated, real sequences or when the number of base velocities are increased (then several spurious models may be present) the 
prominent models are obtained by increasing this parameter, $c \in[1,4]$. Note also that in all cases, we compute our dense optical flow using at most 200 iterations.

\section{Experiments on Synthetic and Real Sequences}

Local Measurements Are Noise Sensitive. Figure 1(a) shows a synthetic sequence (size $54 \times 54 \times 16$ ) with transparent motion, similar to the one in [14]: There is a moving background (with velocity $\hat{u}=[0,-1]$ ) and an overlapped moving transparent square (with velocity $\hat{v}=[1,0]$ ), with additive Gaussian noise. Figure (b) (resp. (c)) show the OFs associated to the minima of distance (11) (resp. (2)). This represents indeed what will be the input of our approach and illustrate the need of velocity integration.

Regularization of local measurements. Sequence in Figure1(a) is corrupted with Gaussian noise to measure the robustness of our approach. Figures 2 (a)-(c) show the results obtained. The percentages of pixels with a wrong estimation are $2.12 \%, 2.29 \%$ and $2.40 \%$ respectively: Our approach can deal with a strong noise corruption, with better results than [14] (see Figures 2 (d) and 20 (e), and results in 14). The velocity basis was composed by 33 vectors, specified through their magnitudes and orientations, respectively $\{0,1,2,3,4\}$ pixels and $\left\{0, \frac{\pi}{4}, \frac{\pi}{2}, \frac{3}{4} \pi, \pi, \frac{5}{4} \pi, \frac{3}{2} \pi, \frac{7}{4} \pi\right\}$ radians. For comparison purposes, Figures 2 (d) and 2 (e) show the computed OF with the computationally expensive Gibbs sampler approach for minimizing the discrete energy function reported in [14. In [14] a deterministic relaxation ICM algorithm was used, which, differently to Gibbs sampler approach, is prone to converge to local minima. The noise-free case is shown in Figure 2 (d), and the $\mathrm{SNR}=30$ case in Figure 2 (e). The results shown correspond to the computed solution after 150,000 iterations (about 2.5 hours, in a PC Pentium IV, 3.0 GHz) that represent 150 times slower than our approach. For the Gibbs sampler results, the quality decreases for noise corrupted sequences (see Figure 2 (e) and compare it with the one computed with the proposed method in about 1 minute in Figure 2 (f)). The behavior of the spatial regularization and intra-model competition is illustrated in Figure 3 . It shows the evolution of the layer associated with velocity [1,0]: Large values appear in the square region whereas small values appear in the background region.

Realistic Textured Sequences. High textured sequences are relatively easy to solve using local motion measures. The real performance of the method should be evaluated in realistic textured scenes, where we may find difficulties because several models may locally explain the data. The next experiment is composed of two moving photographs: a face $I_{1}$ with motion $u=[1,0]$ (limited textured scene) and a rocky Mars landscape $I_{2}$, with motion $v=[-1,0]$. The sequence was generated with $f=0.6 I_{1}+0.4 I_{2}$, see Figure 4 (a).

Figure 4 (b) shows the OF associated to the minimum distance (2) used in the attach term. The computed velocity field is shown in Figure 4 (c). Note that the right $\mathrm{OF}$ is recovered in all the pixels regardless of the high amount of noise. 


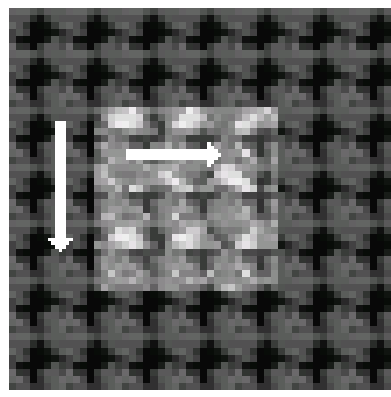

(a) Noise free sequence

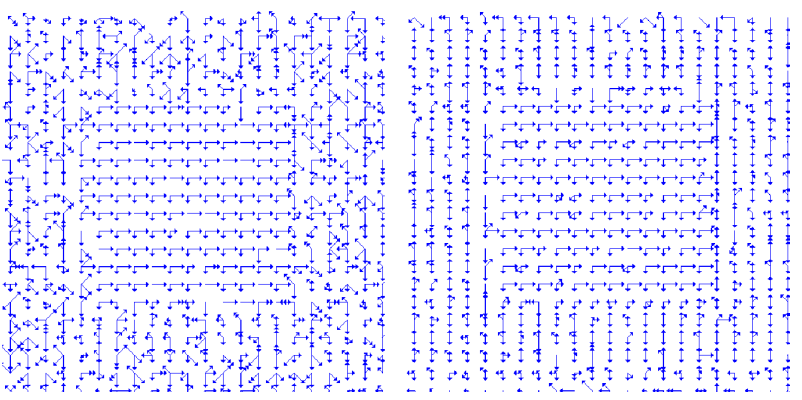

(b) OFs from $d_{\mathcal{C}}$

(c) OFs from $d_{\mathcal{D}}$

Fig. 1. Synthetic noisy sequence $(\mathrm{SNR}=30)$ and results obtained with the distances $d_{\mathcal{C}}$ (1) and $d_{\mathcal{D}}$ (2)

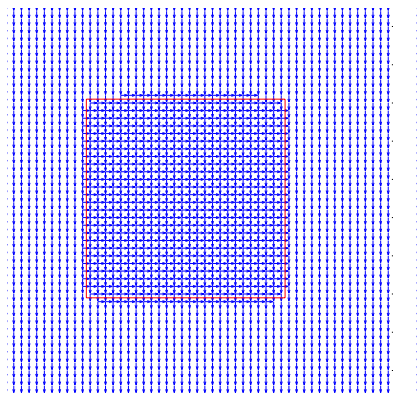

(a) $\mathrm{SNR}=30$

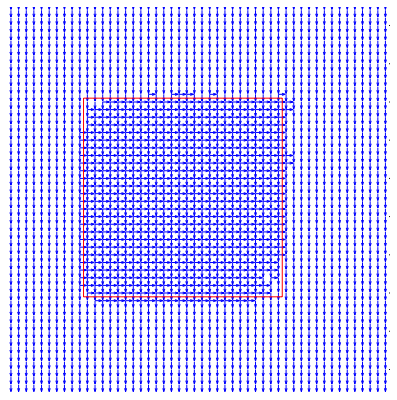

(b) $\mathrm{SNR}=10$

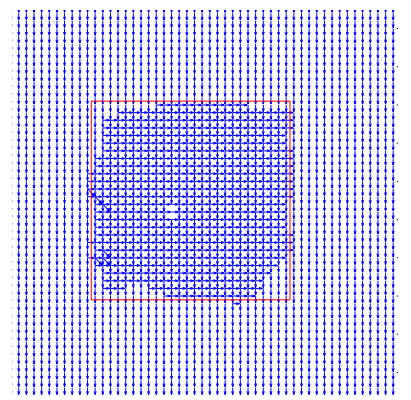

(c) $\mathrm{SNR}=6.5$

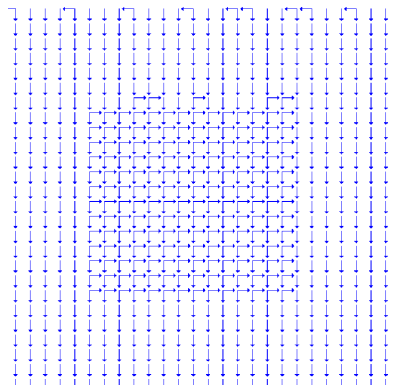

(d) Gibbs S., noise-free

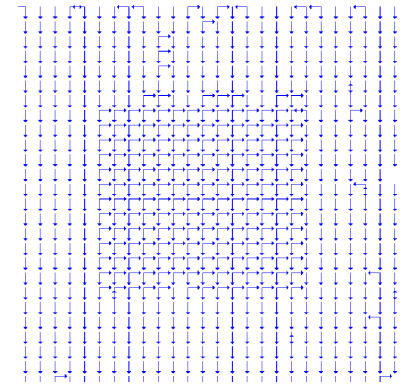

(e) Gibbs S., SNR=30

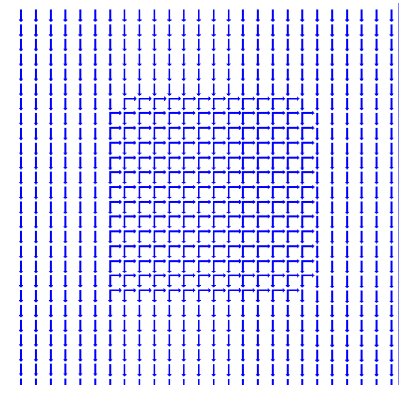

(f) Our result, $\mathrm{SNR}=30$

Fig. 2. First row. Results obtained with our approach applied to the synthetic sequence presented in Figure 1 (a) with different noise levels (input was $d_{\mathcal{C}}$ ). Second row. Comparison with Gibbs Sampler scheme: (d) noise-free case and (e) noisy case $\mathrm{SNR}=30$. (f) our result for $\mathrm{SNR}=30$, confront with (e). 

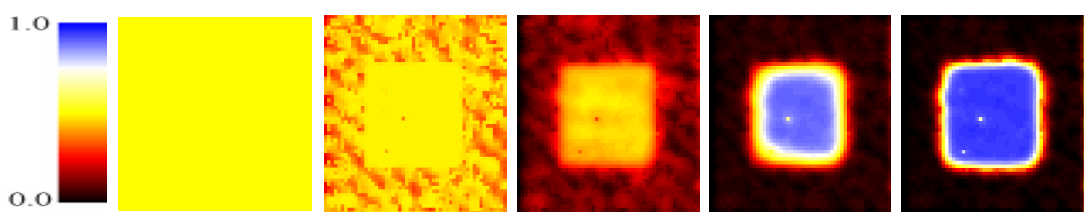

Fig. 3. Evolution during minimization (in pseudo-color scale) of $\alpha_{i}(r)$ for the velocity $u_{i}=[1,0]$ for a strongly noise corrupted sequence $(\mathrm{SNR}=15)$. Iterates $0,1,11,31$ and 200 are shown.

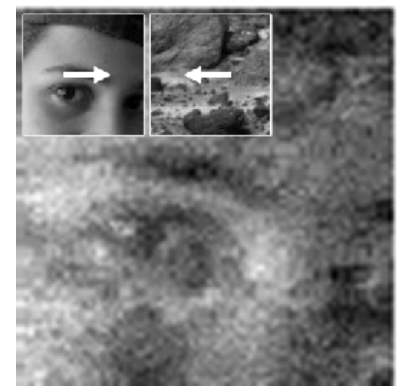

(a) Noisy sequence $(\mathrm{SNR}=8)$

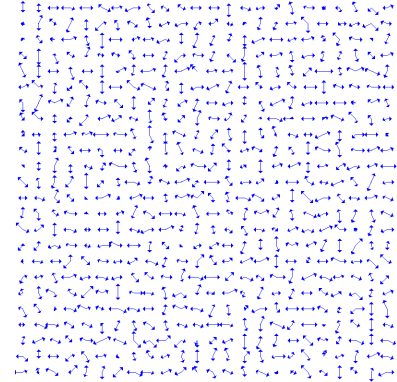

(b) OFs from $d_{\mathcal{D}}$

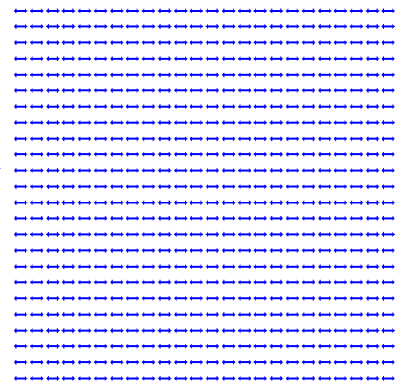

(c) Our result

Fig. 4. Two noisy textured patterns in translation. We show the velocities associated to minimum distance (2) and our regularized result.
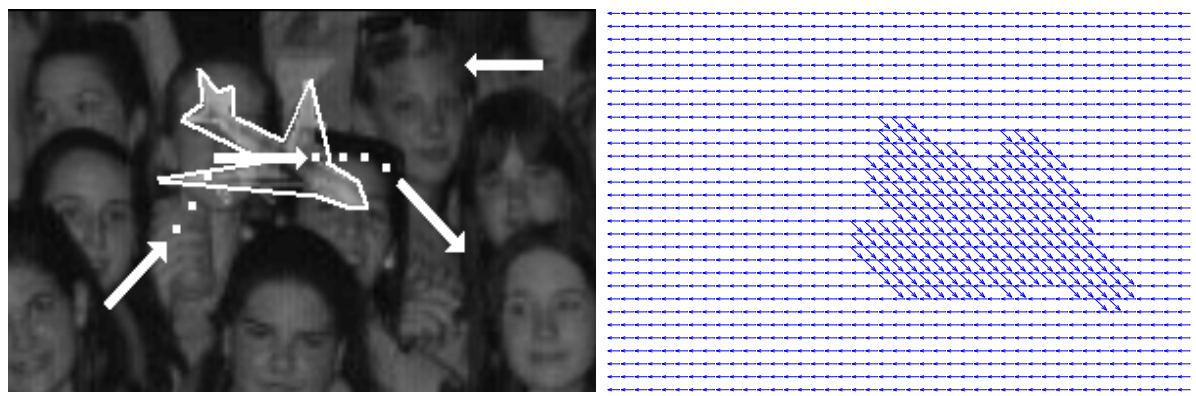

Fig. 5. Transparent object moving with changing translational speed over a translating background and one of the recovered motion fields

Figure 5 shows a sequence with a time-varying transparent region and motions. The changing velocities are schemed in Figure 5 (a). An example of the obtained multi-velocity vector field is shown in Figure 5 (b) (results for more frames of this sequence can be found in [23). For this experiment we used the distance measure in (11).

Figure 6 shows results for a rotating layer sequence. The sequence was made by adding a rotating earth globe (1 degree per frame) and a translating textured 


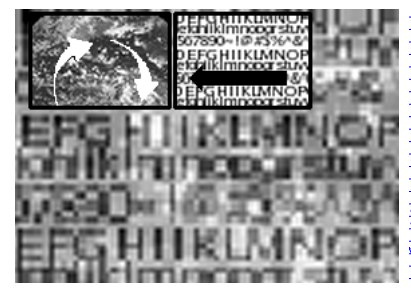

Sequence

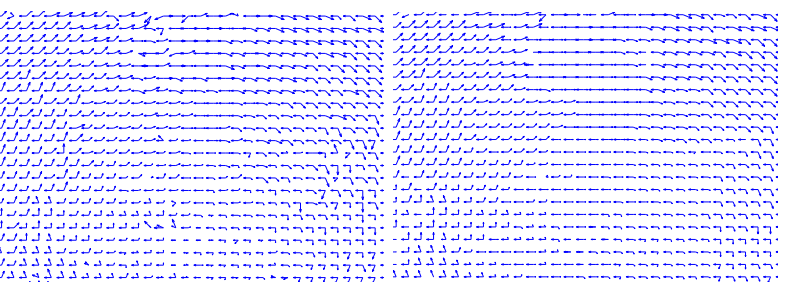

OFs from $d_{\mathcal{C}}$
Our result

Fig. 6. Transparent motion sequence with complex rotating motion, $\mathrm{SNR}=60$. We show the sequence, associated velocities to minimum distances (1) and our method's result.
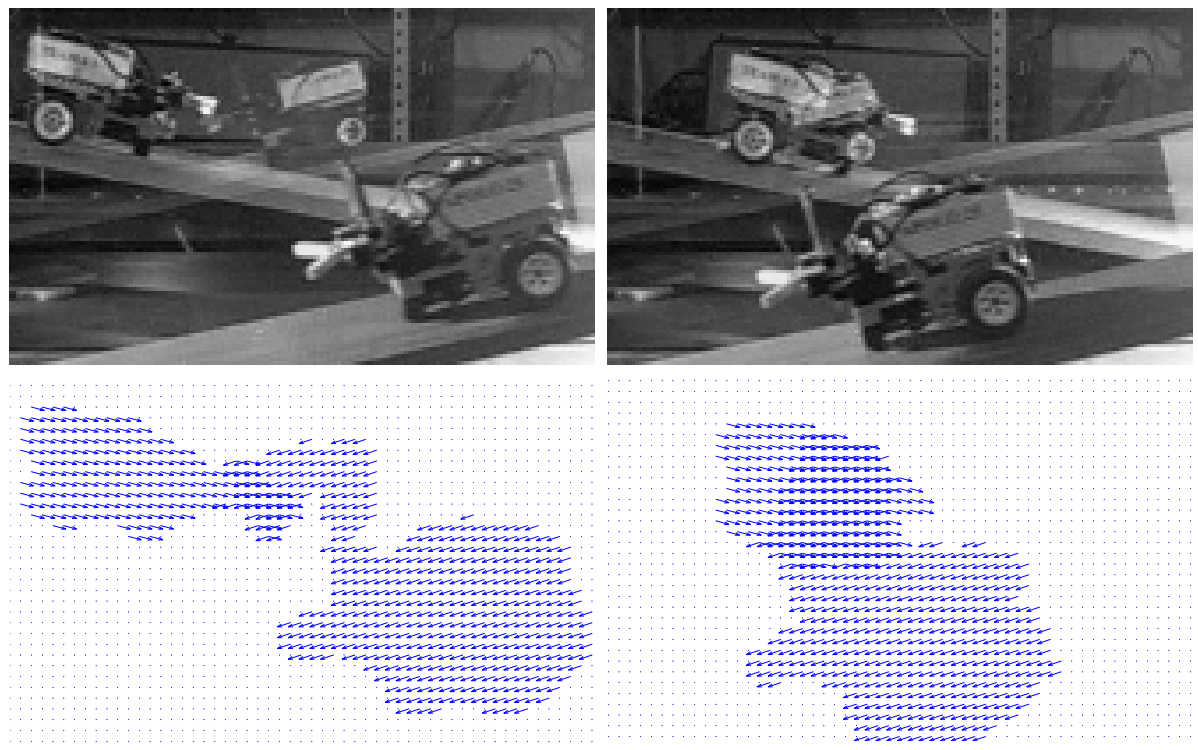

Fig. 7. Real transparent sequence and our results in the second row

image with velocity $[-1,0]$, as it is indicated in Figure 6 (a). Note that in such a case a large set of velocities (either different in orientation and magnitude) are computed as the final solution in Figure 6 (c). In this experiment we estimate dense smooth flow which does not rely on any motion assumption or model.

Transparency and Occlusion in a Real Sequence (Figure 7). We show the obtained results for a real sequence composed by two robots moving down a slope. The upper-left robot is located behind a glass while the lower-right robot is located in front of the camera. The reflection of the second robot is located in the upper-central part. For this experiment we used as input the distance measure (1). The recovered velocities were $[1.5,0.4]$ pixels for the upper-left robot and $[-1.5,0.5]$ for both the lower-right robot and its reflection, see second row in 
Figure [7. Despite the fact that the lower right-robot is moving a little faster than its reflection (easy to deduct form the projection geometry), both are detected with the same velocity model. That is explained by the low resolution of the discrete velocity basis. For this experiment, we perform a spatiotemporal Gaussian smoothing process $(\sigma=0.5)$ of the sequence and we process only the regions that contain displacements: the static background was removed automatically by thresholding the difference between consecutive frames, and then applying opening-closing morphologic operators.

\section{Conclusion}

We have presented a novel variational formulation for the estimation of multiple motions, and especially transparency. The unknown is a vector valued field that indicates the presence of some given motions at each spatiotemporal location. Our formulation extends previous works based on layered OF computation, by using a distance measure suitable for transparent motions and proposes an intra-model competition mechanism well-suited for multi-valued solutions. In our case, multiple motions, the intra-model competition behaves similar to the mechanisms used for entropy-control for single motion fields. This term is by itself a novel contribution of this work, since we do not need special preprocessing in order to tackle sequences with one or more layers, as was shown in synthetic experiments in textured and non-textured sequences as well as real sequences.

In future extensions of this work, we will study in more depth the diffusion terms and also investigate how the different velocity maps may interact together. We also want to evaluate our approach on test sequences used in psychophysics, which will certainly suggest some improvement of the current model.

\section{Acknowledgments}

A. Ramirez-Manzanares and M. Rivera were partially supported by CONACYT, Mexico, PhD Scholarship and grant 46270, respectivelly.

\section{References}

1. Oppenheim, A.V.: Superpostion in a class of nonlinear systems. In: IEEE International Convention, New York, USA (1964) 171-177

2. Bergen, J., Burt, P., Hingorani, R., Peleg, S.: Computing two motions from three frames. In: ICCV 90, Osaka, Japan (December 1990) 27-32

3. Burt, P., Hingorani, R., Kolczynski, R.: Mechanisms for isolating component patterns in the sequential analysis of multiple motion. In: IEEE Workshop on Visual Motion, Princeton, NJ (October 1991) 187-193

4. Irani, M., Peleg, S.: Motion analysis for image enhancement: resolution, occlusion, and transparency. Journal on Visual Communications and Image Representation 4(4) (1993) 324-335

5. Irani, M., Rousso, B., Peleg, S.: Computing occluding and transparent motions. IJCV 12(1) (January 1994) 5-16 
6. Shizawa, M., Mase, K.: Simultaneous multiple optical flow estimation. In: ICPR 90. Volume 1. (1990) 274-278

7. Shizawa, M., Mase, K.: Principle of superposition: a common computational framework for analysis of multiple motion. In: IEEE Workshop on Visual Motion. (1991) $164-172$

8. Shizawa, M., Mase, K.: A unified computational theory for motion transparency and motion boundaries based on eigenergy analysis. In: CVPR 91. (1991) 289-295

9. Liu, H., Hong, T., Herman, M., Chellappa, R.: Spatio-temporal filters for transparent motion segmentation. In: ICIP 95. (1995) 464-468

10. Darrell, T., Simoncelli, E.: Separation of transparent motion into layers using velocity-tuned mechanisms. In: MIT Media Laboratory Vision and Modeling Group Technical Report. Number 244 (1993)

11. Mota, C., Stuke, I., Aach, T., Barth, E.: Divide-and-Conquer strategies for estimating multiple transparent motions. In: 1st International Workshop on Complex Motion, Schloss Reisensburg, Germany. LNCS Vol. 3417. (2005) 66-78

12. Mühlich, M., Aach, T.: A theory of multiple orientation estimation. In: ECCV. Volume 2. (2006) 69-82

13. Stuke, I., Aach, T., Barth, E., Mota, C.: Estimation of multiple motions by block matching. In: 4th ACIS International Conference on Software Engineering, Artificial Intelligence, Networking and Parallel/Distributed Computing (SNPD 2003). (2003) 358-362

14. Stuke, I., Aach, T., Barth, E., Mota, C.: Multiple-motion-estimation by block matching using MRF. In: ACIS, International Journal of Computer and Information Science. (2004)

15. Auvray, V., Bouthemy, P., Lienard, J.: Motion estimation in x-ray image sequence with bi-distributed transparency. In: ICIP 06, Atlanta, USA (2006)

16. Fitzpatrick, J.: The existence of geometrical density-image transformations corresponding to object motion. CVGIP 44(2) (November 1988) 155-174

17. Black, M., Anandan, P.: The robust estimation of multiple motions: Parametric and piecewise-smooth flow fields. CVGIP: Image Understanding 63(1) (1996) 75-104

18. Jepson, A., Black, M.: Mixture models for optical flow computation. In: CVPR 93. (1993) 760-761

19. Ju, S., Black, M., Jepson, A.: Skin and bones: Multi-layer, locally affine, optical flow and regularization with transparency. In: Proceedings of CVPR 96, San Francisco, CA (June 1996) 307-314

20. Black, M., Fleet, D., Yacoob, Y.: Robustly estimating changes in image appearance. Computer Vision and Image Understanding 78 (2000) 8-31

21. Weiss, Y., Adelson, E.: A unified mixture framework for motion segmentation: incorporating spatial coherence and estimating the number of models. In: CVPR 96. (1996) 321-326

22. Rivera, M., Ocegueda, O., Marroquin, J.L.: Entropy controlled gauss-markov random measure field models for early vision. In: VLSM 05, LNCS 3752. (2005) 137-148

23. Ramirez-Manzanares, A., Rivera, M., Kornprobst, P., Lauze, F.: Multi-valued motion fields estimation for transparent sequences with a variational approach. Technical Report RR-5920, INRIA, (Also, Reporte Técnico CIMAT, (CC)I-06-12) (June 2006)

24. Black, M., Rangarajan, P.: On the unification of line processes, outlier rejection, and robust statistics with applications in early vision. IJCV 19(1) (1996) 57-91

25. Ramirez-Manzanares, A., Rivera, M.: Brain nerve boundless estimation by restoring and filtering intra-voxel information in DT-MRI. In: VLSM 03. (October 2003) $71-80$ 